\title{
Heike Krieger \\ Fehlgeschlagene Dekolonialisierung \\ Grenzen von Anwendbarkeit und Durchsetzbarkeit des Selbstbestimmungsrechts der Völker am Beispiel Ost-Timors
}

Das Prinzip des Selbstbestimmungsrechts der Völker hat, abstrakt formuliert, allgemeine Geltung. Sobald aber das Prinzip in seinem tatsächlichen Anspruch konkretisiert wird, beginnen Kontroversen in der Völkerrechtslebre. Dies zeigt exemplarisch der Fall von Ost-Timor. Das Selbstbestimmungsrecht gilt als ungeeignet für die Konfliktregulierung.

Die Red.

\section{Der Fall Ost-Timor}

Die Ost-West Teilung der Insel Timor reicht in dic Epoche der Kolonialisierung zurück. Im achtzchnten Jahrhundert beanspruchte Portugal die östliche Hälfte Timors als Kolonie, während die Niederlande ihre Macht im Westen festigten.' 1949 wurde West-Timor Teil des unabhängigen Staates Indonesien. Die Generalversammlung (GV) der Vercinten Nationen (VN) verlich Ost-Timor 1960 in der Resolution I 542 (XV) vom 15. I2. 1960 den Status eines Hoheitsgebietes ohne Selbstregierung nach Kap.XI, Art. 73 der Satzung der Vercinten Nationen (SVN). Portugal aber behandelte Ost-Timor in den folgenden Jahren weiterhin als Tcil scincs Staatsgebietes und vernachlässigte seine Kolonie in crhcblichem Umfang, so daß die Entwicklungsziele des Art. 73 SVN nicht erreicht wurden. ${ }^{2}$ Die VN verurteilten in cincr Reihe von Resolutionen die portugiesische Vorgehenswcise. ${ }^{3}$

Nach der Nelkenrevolution 1974 änderte die portugiesische Regierung ihre Kolonialpolitil. Sie erließ zwei Gesetze mit Verfassungsrang, in denen sic das Selbstbestimmungsrecht und das Recht auf Unabhängigkeit der Gebiete unter portugicsischer Verwaltung formell anerkannte. ${ }^{4}$ Daraufhin bildeten sich in Ost-Timor verschiedene politische Gruppicrungen. Die UDT (Uniao Democrátıca de 7ïmor) trat für eine Förderation mit Portugal ein, die allmählich zur Unabhängigkcit führen sollte. Die politisch linksorientierte Gruppierung FRETILIN (Frente Revolutionára Timor Leste), dic dic weitrcichendste Unterstützung in der Bcvölkerung fand, strebte nach

1 Defert, Timor Fst, Le Génocide Oublé (1992), S. 3 r; Lawrence, in: Bernhardt (ed.), EPIL, Instalment 12 (1990), S. 94 (95).

2 Art. 73 SVN steht cinen eigenen volkerrechtlichen Status fuir Kolonien vor. Das Gebiet wird von dem der Kolonıalmacht abgesondert und gilt als volkerrechtlich eıgenes Territorium; Fastenrath, in: Simma (Hrsg.), Charta der VN, Kommentar (1991), Rdnr. 1; 1974 7.. B. waren $90 \%$ der Bevolkerung Ost-Timors Analphabeten; A/roo23/Add. 1, 20.11.1975, para.55.

3 U.a. A/Res/1699 (XVI) v. 19.12.1961, 1807 (XVII) 4. 14.12,1962 und schließlich 3294 (XXIX) v. 13.12.1974, in der die VN die Anderung der portugiesischen Kolontalpolitik begrußen.

4 Diario do Governo, 27. 7. 1974, 1. Serie Nr. 174 und Diario do Governo, 17. 7.1975, 1. Serie Nr. 16\}, abgedr. bes: Krieger, East Timor and the international Community (1997), S. 34. 
ren Gibraltar'3, die Westsahara', Eritrea's, mit Einschränkungen auch Palästina ${ }^{16}$ sowie die Falkland-Inseln'7.

In tatsächlicher Hinsicht gibe es einige Parallelen. Insbesondere ist die Situation in der Westsahara und in Ost-Timor wegen ihrer bis in die siebziger Jahre dauernden kolonialen Vergangenheit als spanische bzw. portugiesische Kolonie vergleichbar. Beide Kolonialmächte waren in dieser Zeit grundsätzlich bereit, die Dekolonialisierung durchzuführen, befolgten ihre Pflichten aber nicht konsequent. Die jerveiligen Nachbarstaaten, Marokko und Indonesien, machten territoriale Ansprüche auf das Gebiet geltend und setzten schließlich militärische Gewalt ein, um die faktische Lage zu ihren Gunsten zu verändern. Hierdurch wurden dic Dekolonialisierungsprozesse abrupt unterbrochen. Auch der geopolitische Rahmen ist ähnlich, da beide Gebicte wirtschaftlich und strategisch bedeutend sind. Die Küstenlage vermag einen Anspruch auf eine ausschließliche Wirtschaftszone zu begründen. ${ }^{18}$ Die USA und andere westliche Staaten unterstützen sowohl Indonesien als auch Marokko. ${ }^{19}$

Auch auf der rechtlichen Ebenc ist die Argumentation in allen Fällen ähnlich. Während sich die eine Konfliktpartei darauf beruft, daß die Dekolonialisierung zur Unabhängigkeit ihres Volkcs führen soll, verlangt die andere Seite - zumeist der politisch mächtigere benachbarte Staar, der historische Titel über das Gebiet beansprucht - cbenfalls auf Grundlage der Ausübung des Selbstbestimmungsrechts dic Integration in ihr Gebiet. ${ }^{20}$ Die Konflikte verdeutlichen das Spannungsverhältnis zwischen den Prinzipien der territorialen Integrität und der Selbstbestimmung.

\section{Der rechtliche Gehalt des Selbstbestimmungsrechts der Völker}

Das Selbstbestimmungsrecht beansprucht in der gegenwärtigen Völkerrechtsordnung rechtliche Geltung und stcllt nicht nur einen politischen Programmsatz dar. ${ }^{21}$ Der Internationale Gerichtshof (IGH) hat die Bedeutung des Sclbstbestimmungsrechts als eines der wesentlichen Prinzipien des modernen Völkerrechts mit ciner Wirkung erga omnes bestärigt. ${ }^{22}$ Aus der historischen Entwicklung des Selbstbestim-

13 Cassese, Self-Decermination of Peoples (1995), S. $206 \mathrm{f}$.; die VN fuhren Gibraltar in der Liste der Hoheitsgehiete ohne Selbstregierung nach Art. 73 SVN; vertiefend: Levie, The Staus of Gibraltar (1983); Morris/Haigh, Britain, Span and Gibraltar (1991).

14 Vertiefend: Okere, ICLQ 28 (1979), S. 296-312; Riedel, GYIL 19 (1976), S. 405-442.

is Der Einsatz der VN fuhrte zu einer Losung des Konflikts um Eritrea. Das Land wurde nach Durchfuhrung eines Referendums unter VN-Úberwachung am 27.4.1993 unabhangig; AdG 1993. 3786. I.; vertiefend Goy, A.F.D.I. 39 (1993), S. 337-356; Peters, Das Gebietsreferendum im Volkerrecht (1995), S. $225-246$.

16 Bowring, in: CIIR (Hrsg.) (Fn. 12), S. 165 ; Cassese (Fn. 13), S. 206 sicht die Vergleschharkett mit der Situation Ost-Timors in dem Anspruch auf Selbsthestimmung gegenuber Fremdherrschaft; Hilpold, Der Osttımor-Fall (1996), S.7t.

17 Siche hierzu Franck, New York University Journal of International Law and Politics 8 (1975), S. 331 (379-384); auch die Falkland-Inseln werden auf der Liste der Hoheitsgebiete ohne Selbstregierung nach Art. 73 SVN gefuhrt.

18 Franck (Fn. 17), S. 334

19 Hilpold (Fn. 16), S. 71 ; Rigaux, in: CIIR (Hrsg.) (Fn. 12), S. 167 f.

20 Cassese (Fn. 13), S. 206/209/214/221/235; Franck (Fn. 17), S. 334/369. Die Argumentation wird auch im Fall Palastınas vorgebracht. Beıspıelsweise beruft sich Israel darauf, daß die arahıschen Palastinenser ihr Selbstbestımmungsrecht mit Grundung Jordanıens ausgeubt hatten; Stellungnahme Yehuda Blums vor den VN 1980, GAOR, XXXVth Session, Plenary Meetings, 77th Meetıng, 2.12.1980, S. 1318 paras. 108-109.

21 Cristescu, Self-Determination, E/CN. $4 /$ Sub. $2 / 404 /$ Rev. 1, S. 23 f., para. 154 ; E. Klein, Das Selbstbestummungsrecht der Volker und die deutsche Frage (1990), S. 35; Pomerance, Self-Determination in Law and Practice: The New Doctrine in the United Nations (1982), S. 71 ; ablehnend: Partsch, in: Wolfrum (Hrsg.), Handbuch Vereinte Nationen (1991), S. 747 Rdnr. I1.

22 Opinion on the Legal Consequences for States of the Contınued Présence of South Africa in Namibıa 
sofortiger Unabhängigkeit. APODETI (Associacao Popular Democratica de Timor) trat für eine Vereinigung mit Indonesien ein.' Eine Übergangsregierung und ein portugiesischer Hochkommissar sollten allgemeine, geheime, gleiche und unmittclbare Wahlen zu einer Volksversammlung, die über die Unabhängigkeit Ost-Timors hätte entscheiden müssen, für den Oktober 1976 vorbereiten.

Im August 1975 brach ein Bürgerkrieg zwischen den ost-timoresischen Partcien aus, auf Grund dessen dic portugiesische Verwaltung die Kontrolle über das Gebiet verlor. Am 28. x I. 1975 gab FRETILIN, das den größten Teil des Territoriums kontrollierte, eine einseitige Unabhängigkeitserklärung ab. ${ }^{6}$ Kurz darauf erklärte eine Koalition der anderen Bürgerkriegspartcien die Unabhängigkeit Ost-Timors und dic gleichzeitige Vereinigung mit der Republik Indonesien.7 Am 7. I 2. 1975 marschierten indonesische Truppen in Ost-Timor ein. Am 31.5. 1976 trat eine Volksversammlung in Dili zusammen, die sich für die Vereinigung mit Indonesien aussprach. ${ }^{8}$ In einem formalen Akt am 17.7.1976 beschloß das indonesische Parlament den Beitritt OstTimors als 27. Provinz Indonesiens. ${ }^{9}$

Dic militärischen Auseinandersetzungen zwischen Indonesien und FRETILIN hielten bis $1978 / 79$ an. Bis 1984 sollen 200000 der 650000 Einwohner Ost-Timors in militärischen Auseinandersetzungen mit der indonesisehen Armee sowie durch Epidemien und Hungersnöte zu Tode gekommen sein. ${ }^{10}$ 1985 initiierte die VNMenschenrechtskommission das vertrauliche Verfahren der Resolution is 03 des Wirtschafts- und Sozialrates. "Weite Teile der Bevölkerung leisten bis heute aktiven oder passiven Widerstand gegen die Einverleibung. ${ }^{12}$

\section{Unbewältigte Dekolonialisierungsprozesse}

Einige andere der ungelösten Konflikte der Gegenwart spiclen sich vor einem vergleichbaren Hintergrund ab, auch wenn die Fälle fehlgeschlagener Dekolonialisierung jeweils erhebliche historische Besonderheiten aufweisen. Zu Beispielen solcher Konflikte, die die eigentliche Phase der Dekolonialisierung überdauert haben, gehö-

$5 \mathrm{~A} / 10023 / \mathrm{Add} .1,20.11,1975$, paras. 11-16.

6 AdG 1976, 20306. Albanien, Benin, Angola, Guinea, Guinea-Bissau, Kambodscha, Kap Verde, Laos, Mozambique, Nordkorea, Sao Toné und Princıpe, Tansania, Viernam, die Volksrepublik Chuna und die Volksrepublık Kongo erkannten die Demokratische Republik Ost-Timor an, z. B. Stellungnahme des Vertreters Mozambiques, SCOR, 31st Year, 1912th Meeting, 20.4. 1976, para. is.

7 Joint Proclamation by APODETI, UDT, KOTA and the Partido Trabilhisca, Batugade 30. 11. 1975, A.C. $4 / 808$.

8 Report of the Secretary-General in Pursuance of Security Councll Resolution 389 (1976), S/12106, Annex, 22.6. 1976 , paras. $4 / 6$.

9 AdG 1976, 20307/20389.

10 The Europa World Year Book 1994 , Vol. 1/2, S. 1463 (1465); Lawrence (Fn. 1), S. 95 .

$11 \mathrm{E} / \mathrm{CN} .{ }_{4} /$ I $985_{5} / \mathrm{SR} .{ }_{4}$ s/Add. I, S. 2 para. 1 ; das Verfahren zielt nicht auf die Prufung von Verlutzungen im konkreten Fall, sondern auf die Emittlung eines Gefleches systematiwh begangener, schwerer und zuverlassig bezeugter Minschentechssverleczungen.

12 Commission on Human Rights, Report of the Secretary-General on the Situation in East limor, 31. 1. 1995, E/CN. 4/1995/72; Aditiondro, in: CIIR (Hrsg.), International Law and the Question of East Timor (1995), S. 50 (53); anlaßlich der Parlamentswahlen un Indonesien kam es am 29. 5. 1997 zu schw'ren Ausschreitungen, die auf Seiten der Timoresen nach Angaben von Reater 7 wischen is und 22 Toten forderten. 
mungsrechts können drei potentielle Rechtsträger hergeleitet werden. Ausgehend vom Beginn der Entwicklung des Rechtes aus dem institutionalisierten Minderheitenschutz im Völkerbund, sind Minderheiten in einem ethnisch fremden Staatsverband Träger des klassischen Selbstbestimmungsrechts der Völker. Weiterhin gehören zu den Rechtsträgern die Kolonialvölker. Schließlich ist aus dem Gedanken der Abwehr von Fremdherrschaft, der dem Selbstbestimmungsrecht immanent ist, der dritte potenticlle Rechtsträger ableitbar: das Staatsvolk eines bestehenden Staates ist Träger des Rechtes, wenn der Gesanitstaat unter Fremdherrschaft, z. B. im Fall der Annexion, gerät. ${ }^{23}$

Angesichts dieser verschiedenen Träger spaltet sich auch der Inhalt des Rechts in unterschiedliche konkrete Ansprüche auf. Sie werden den zwei Anwendungsbereichen des Selbstbestimmungsrechts zugeordnet: Der äußere ist gegen andere Völkerrechtssubjekte gerichtet, und der innere zielt auf die Verhältnisse im Staat. Das inncre Sclbstbestimmungsrecht kann in das Recht zur freien Wahl der eigenen Regicrung, den Minderheitenschutz und die Frage der staatsinternen Demokratie untergliedert werden, während das externe Selbstbestimmungsrecht das Recht auf Nichteinmischung und auf Gründung eines eigenen Staates, insbesondere auf Dekolonialisierung umfaßt. Unbestritten sind Inhalt und Umfang der Ansprüche auf Nichteinmischung und Dekolonialisierung sowie das Recht auf freie Wahl des politischen Systems. ${ }^{24}$ Umstritten aber sind Gehalt und Ausmaß der Rcchte der Minderheiten und der Rechtsnormcharakter des Rechts zur demokratischen Willensbildung im Staat. ${ }^{2 s}$ Das Recht auf Gründung eines eigenen Staates steht jedenfalls Kolonien, Treuhandgebieten und anderen Hoheitsgebieten ohne Selbstregierung i.S. des Art. 73 SVN zu. ${ }^{26}$ Die Begrenzung ist durch die Bedeutung des Grundsatzes der territorialen Integrität der bestehenden Staaten zu rechtfertigen. Demgemäß haben die VN in der Dekolonialisierungsresolution GVRes Is I4 (XV) vom 14. 12.1960 zwar ein Recht auf Unabhängigkeit von Kolonialstaaten, aber kein Recht auf Sezes sion begründet. ${ }^{27}$ In Einklang mit der VN-Praxis kann der Anwendungsbereich des externen Selbstbestimmungsrechts aber auf andere Gebiete ausgedehnt werden, dic geopolitisch abgrenzbare Bereiche bilden und deren Einwohner nicht an der Regierung innerhalb der Region oder des Staates beteiligt sind, so daß sie tatsächlich den Hohcitsgebieten ohne Selbstregierung gleichzustellen sind. Zur Ermittlung letzterer Gruppe kann auf die GVRes IS4 I (XV) vom 15.12. 1960 zurückgegriffen werden. ${ }^{28}$ Mit der Staatenpraxis vereinbar erscheint auch die Annahme, daß das Sezessionsrecht als self-belp remedy zuzubilligen ist, wenn der Sezession Unterdrückung durch

(South West Africa) notwthstandıng Security Council Resolution 276 (1970), I.C.J. Reports 1971 , S. 16 (31 para. 52); Western Sahara, Advisory Opinion, I.C.J. Reports i975, S. 12 (3I-33 paras. 54-59); East Timor, Judgment, I.C.J. Reports 1995, S. 90 (102 para. 29).

23 Dochring nach Art. 1, in: Simma (Fn. 2), Rdnr. 29-31.

24 Doehring (Fn. 23), Rdnr. 32-48.

25 Doehring (Fn. 23), Rdnr. 49-53; Peters (Fn. 15), S. 287-432; vor allem Franck, AJlL 86 (1992), S. $46-91$.

26 Crawford, Creation of States (1979). S. 92-94; fur eine Anwendung jenseits der Dekolonialisierung Dochring (Fn. 23), Rdnr. 48 .

$27 \times 6$. Any attempt aimed at the partial or total disruption of national unity and the territorial integrity of a country is incompatible with the purposes and princpiples of the Charter of the United Nations.... A/Res 1514 (XV) vom 14.12.1960; KSZE-Schlußakte von Helsinıkı, Prinzıp VII, Korb I, Bulletin der Bundesregierung 1975, 968 (970); noch in der Charta von Parıs wird im Rahmen der KSZE die Bedeutung der territorialen Integritat der Staaten betont, EA 1990, D $656(658)$; Halperin/ Scheffer, Self-Determination in the New World Order (1992), S. 74-76; Pomerance (Fn. 21), S. 68.

28 Crawford (Fn. 26), S. 92-94/1 r6f.; S. ror mit dem Beispıel Bangladesh; Dissentung Opinion Judge Fitzmaurice, 1.C.J. Reports 1971 (Fn. 22), S. 16 (296 para. 121), fur Sud-West Afrika; eine zuruckhaltende Bewertung nimmt Higgins, in: Brolmann et al. (Hrsg.), Peoples and Mınorsties in International Lasw (1993), S. 33 vor. 
erhebliche Menschenrechtsverletzungen vorausgegangen ist ${ }^{29}$ oder wenn es um die Wiederherstellung eines früheren, völkerrechtswidrig beseitigten Territorialzustandes geht. ${ }^{30}$ Nachdem der Dekolonialisierungsprozeß weitgehend abgeschlossen worden ist, steht heute wiederum dieselbe Ausgangsfragestcllung wie zu Beginn dieses Jahrhunderts nach der Rolle der Minderhciten und der Grenzen eines Rechts zur Sczession im Zentrum des Selbstbestimmungsrechts."

\section{Fehlende Anwendbarkeit des Rechtsprinzips oder mangelnde Durchsetzbarkeit des Anspruchs in der Staatengemeinschaft?}

Obwohl das Recht auf Selbstbestimmung von Kolonien also allgemein ancrkannt ist, ist es verwunderlich, daß einige der schwelenden politischen Konflikte gerade ehemalige Kolonien betreffen, in denen eine Konfliktlösung mittels rechtlicher Instrumentc bisher nicht erreicht wcrden konnte. Die Erklärungen für dieses Phänomen sind unterschiedlich. Kritische Stimmen führen die Diskrepanz auf Mängel in der herrschenden Konzeption des Selbstbestimmungsrechts der Völker bci der Abwägung mit dem Grundsatz der territorialen Integrität der Staaten zurück, ${ }^{32}$ während andere auf den fehlenden politischen Willen abstellen. ${ }^{34}$

Die portugiesische Völkerrechtlerin Escarameia erläutert am Fall Ost-Timors konzeptionclle Mängel des Sclbstbestimmungsrechts. Definitionsansätze griffen vor allem auf die historische Entwicklung des Rechts zurück und orientierten sich an der oft auf den Einzelfall bezogenen Staatenpraxis der VN. Zugleich sei diese inhaltliche Auslegung eng verknüpft mit den politischen Vorstellungen der Rechtsanwender. So habe dic sowjetische Völkerrechtslehre das Selbstbcstimmungsrecht als logische Folge des Marxismus-Leninismus in Abgrenzung zum bürgerlichen Prinzip der Nationalstaaten beschrieben ${ }^{34}$, während Autoren aus Ländern der Dritten Welt das Selbstbestimmungsrecht der Völker auf die Dekolonialisierung hätten beschränken wollen. ${ }^{35}$

Die Unsicherheit in der Anwendung des Selbstbestimmungsrechts resultiert laut Escarameia daraus, daß trotz der Einzelfallsituationen versucht werde, das Konzept unter dem Gedanken der Frcihcit von Frcmdherrschaft zu gencralisieren. Es sei aber zu bezweifcln, daß diese Gemcinsamkeit ausrciche, die Geltung eines einheitlich anwendbaren Rechtsprinzips zu begründen, ${ }^{36}$ Durch die in der Literatur weitverbrcitete Aufgliedcrung in mehrere unterschiedliche Ansprüche führten die Autoren

29 Hilpold (Fn. 16), S.89; Thuirer, in: Neuhold/Sımma (Hrsg.), Neues curopaısches Volkerrecht nach dem Ende des Ost-West-Konfliktes, S. 43 (so); fur cin Sezessionsrechr, wenn der Mutterstaat die Arspruche cines Volkes auf scinc kulturellen Rechee und seine Autonomic nicht erfïllt, Murswiek, in: Tomuschat (Hrsg.), Modern Law of Self-Determination (1993), S. 38.

30 Cassese (Fn. 13), S. 90; Thürer (In. 29), S. 43 (45); zu den Fallen, in denen die Staatenpraxis in diesem Kontext Bezug auf das Sclbstbestimmungsrecht genommen hat, gehoren Afghanistan (z. B. Parliamentary Under-secretary of State, ICCO, HC Debs., vol.93, col.1214: 13.3.1986, BYIL 57 (1986!. S. 514) und die Baltischen Staten (Minister of State, FOC, HL. Debs., vol. s02, coll. 179: 29.11. 1988, BYIL 59 (1988), S. 447).

31 Vertiefend Oeter, ZaoRV SI (1992), S. 74 I-780.

32 Cassese (Fn. 13), S. 206; Escaramcia, Formation of Concepts in International Law (1993), S. $92-94 / 110$ f. Ofuatey-Kodjoc, The Principle of Self-Determinacion in International Law (I 977). S. VII; auf dic rechtlichen Scbwierigkeiten der Anwendung im Einzelfall weist auch Koskenniemi, ICLQ 43 (1994), S. 241 (264) hin

33 U.a. Bowring (Fn. 16), S. 163 f.; Hilpold (Fn. 16), S. 78

34 Z. B. Tunkin, Volkerrecht der Gegenwart (1963), S. 36

3s Zu dieser Diskussion: Umozurike, Self-Determination in International Law (1972), S. 197

36 Escarameia, in: CIIR (Hrsg.) (Fn. 12), S. 126 f.; Escarameia (Fn. 32), S. 70. 
unter einem Namen sehr unterschiedliche Fällc zusammen. Damit setzten sie sich der Gefahr des Zirkelschlusses aus, daß das Selbstbestimmungsrecht so unterschiedliche Fälle erfasse, daß nicht mehr von einem Rechtssatz gesprochen werden könne. Zudem sei das Selbstbestimmungsrecht aus der politischen Sphäre entnommen, ohne hinreichend rechtlich durchdacht gewesen zu scin. Bis heute sei die Interpretation des Selbstbestimmungsrechts der Völker von einer Vermischung des nationalistischen Gedankens der Entstehung von Nationalstaaten, wie er in der Entwicklung des 19. Jahrhunderts zum Ausdruck kam, mit dem individualistischen, von Rousseau abzuleitenden Streben nach der ursprünglichen Eigenheit eines Volkes bestimme. ${ }^{37}$ Daher sei das Selbstbestimmungsrecht eine abstrakte Regel mit einem Grad hoher Allgemeinheit, das ohne viel Nutzen zur Lösung konkreter Streitfälle geblieben sei. ${ }^{3}$ Gerade im Fall Ost-Timors hätten die unterschiedlichen Bedeutungen, die dem Begriff des Selbstbestimmungsrechts zugemessen wurden, und die nur vage Definition in der SVN den Entscheidungsprozeß in den VN erschwert. Wegen der Abstraktheit des Begriffs hätten keinerlei operative Konsequenzen aus dem Selbstbestimmungsrecht der Satzung der Vereinten Nationen gezogen werden können. Indonesien habe eine Bandbreite juristischer Argumentationen zur Verfügung gehabt, um seine Aktivitäten zu rechtfertigen. ${ }^{9}$ Der abstrakte Gehalt der VN-Resolutionen habe jede rechtlich mögliche Assoziation erlaubt und zu einer Verschiebung der Betonung vom politischen Kontext der Selbstbestimmung über humanitäre Fragen hin zu reinen Verfahrensfragen, z. B. den Guten Diensten des Generalsekretärs ${ }^{40}$, geführt. ${ }^{41}$ Auf Grund der vielfältigen Bedeutungen des Selbstbestimmungsrechts und der darausfolgenden Ambivalenz im Gcbrauch sei es für die politisch stärkere Partei leicht gewesen, das Konzept in der Praxis völlig unwirksam zu machen..$^{4^{2}}$

Dieser Interpretation steht die verbreitete Ansicht entgegen, daß der fehlende politische Wille zur Durchsetzung der berechtigten Ansprüche Grund für die Situation in Ost-Timor gewesen sei. Das Prinzip der Selbstbestimmung sei gerade im Dekolonialisierungskontext durch dic Rechtsprechung des IGH hinreichend rechtlich konkretisiert, um ein klares Urteil über die Rechtswidrigkeit des indonesischen Vorgehens zu erlauben. Die fehlende Konfliktlösung in Ost-Timor sei allein auf ein politisches Versagen der Staatengemeinschaft zurückzuführen. ${ }^{\text {t3 }}$

\section{Die Bedeutung des Selbstbestimmungsrechts im Fall Ost-Timor}

\section{Völkerrechiswidrigkeit der Invasion und "Inkorporation"}

Indonesien behandelt Ost-Timor als eigenes Staatsgebiet, während Portugal am Fortbestand seiner Verwaltungshoheit festhält. Die indonesische Regierung hat die Invasion und "Inkorporation « als einen Vorgang der freiwilligen Vereinigung dargestellt, den das Volk von Ost-Timor verlangt habe. Zwar beruft sich Indonesien zur

\footnotetext{
37 Koskenniemi (Fn. 32), S. 241 (245f./250).

38 Escarameia, in: CIIR (Hrsg.) (Fn. 36), S. 123

39 Escarameia, in: CIIR (Hrsg.) (Fn. 36), S. 139 ; Escarameia (Fn. 32), S. 103.

40 Unter Guten Diensten werden die Bemuhungen einer Dnttpartci verstanden, die Streitparteien 7u einer friedlichen Losung ihres Streites zu beweyen, z. B. durch die Herstellung von Kontakten oder die Bercitstellung von Moglichkeiten, die Verhandlungen durchzutulıren; sıehe Franck/Nolte, in: Roberss/Kingsbury (ed.), United Nations, Divided World (1993), S. 143.182

41 Escarameia, in: CIIR (Hrsg.) (Fn. 36), S. 141 f.

42 Escarameia, in: CIIR (Hrsg.) (Fn. 36), S. 150

43 U.a. Bowring (Fn. 16), S. 163 f.
} 
Rechtfertigung der Einverleibung Ost-Timors auf das Selbstbestimmungsrecht der Völker, aber eine Überprüfung der Argumentation zeigt, daß Indoncsien nicht nur gegen das Gewalt- und das Interventionsverbot, sondern auch gegen das Selbstbestimmungsrecht verstoßen hat. Die indonesische Vorgehensweise ist völkerrechtswidrig und auf Grund der Verletzung fundamentaler völkcrrechtlicher Normen unwirksam. ${ }^{44}$

\section{a) Die Invasion als Verstoß gegen Gewalt- und Interventionsverbot}

Das allgemeine Gewaltverbot dcs Art. 2 Abs. 4 SVN verbietet jeglichc Anwendung und Androhung bewaffneter Gewalt zwischen den Mitgliedstaaten der VN. Das Gewaltverbot hat sich unter Geltung der Satzung der VN als universelles Völkergewohnheitsrecht ausgebildet. ${ }^{45}$ Es gehört zum Kernbestand der zwingenden Völkerrechtsnormen. ${ }^{46}$ Soweit das Interventionsverbot deckungsgleich mit dem Gewaltverbot die Ausübung militärischer Gcwalt erfaßt, ${ }^{47}$ ist diese Norm ebenfalls cinschlägig. Das Interventionsverbot gilt gewohnheitsrechtlich und hat seinc Grundlage im Prinzip der souveränen Gleich heit der Staaten. Es vcrbietet die Einmischung eines Staates in dic inneren und äußeren Angclegenheiten eines anderen Staates unter Androhung oder Anwendung von Zwang..$^{8}$ Indonesien hat mit dem Einmarsch sciner Truppen unter Ausübung von militärischer Gewalt in ein portugiesisches Hoheitsgebiet ohne Sclbstregierung gegen beide Verbote verstoßen. Dabei ist es nicht erforderlich, auf eine etwaige Staatsqualität der Demokratischen Republik Ost-Timor abzustellen oder sie als de facto-Regime zu qualifizieren. ${ }^{49}$ Jedenfalls gilt Art. 2 Abs. ${ }_{4}$ SVN zwischen Indonesien und Portugal, das als Verwaltungsmacht des Gebiets in eigenen Rechten verletzt worden ist. ${ }^{\circ}$

Indonesien rechtfertigt aber den Einmarsch damit, daß am 30. 11. 1975 alle timoresischen Parteien mit Ausnahme von FRETILIN Indonesien un cine militärische Intervention ersucht haben. Die militärische Einmischung in die inneren Angelegenheiten eines anderen Staates kann zwar ausnahmsweise völkerrechtlich zulässig scin, wenn sie auf Einladung oder mit Zustimmung der Regierung des betroffenen Staates erfolgt. ${ }^{\prime \prime}$ Eine solche Rechtfertigung scheitert aber im Fall Ost-Timors aus mehreren Gründen. Bei einer Intervention auf Einladung im Fall cines Bürgcrkrieges kann zweifelhaft sein, welche Partei berechtigt ist, die Einladung auszusprechen. ${ }^{52}$ Im Fall Ost-Timors ist das Hilfeersuchen nicht vom zuständigen Organ abgegeben worden. Die Verwaltungsmacht Portugal hatte einen entsprechenden Vorschlag Indonesiens abgelehnt. ${ }^{33}$ Im übrigen repräsentierten die koalierenden Bürgerkriegsparteien, die eine Einladung aussprachen, nicht die Mehrhcit der Bevölkerung. Vielmehr benötigte dic Koalition dic indonesische Unterstützung, um cine Übergangsregierung zu bilden und aufrechtzuerhalten. ${ }^{54}$

44 Zur Nichtigkeıt als volkerrechthcher Deliktssanktion: Dorr, Die Inkorporatıon als Tatbestand der Staatensukzession (1995), S, 111-127.

45 Military and Paramilitary Activities in and against Nicaragua, I.C.I. Reports 1986, S. 14 (147).

46 Randelzhofer zu Art. 2 Ziff. 4 Rdnr. 48 , in: Simma (Fn. 2)

47 Fischer, in: Ipsen, Volkerrecht (1990), $\$ 57$ Kdnr. 58.

48 Fischer (Fn. 47), §57 Rdnr. sof.

49 So Hilpold (Fn. 16), S. 68 f.

so Vgl. Jennings/Watts, Oppenheim's International Law, Bd. I/t (1992), \$84, S. 275 ff.; zur Frage des unberechigten Vorwurfs der portugiesischen Derelikuon siehe unter V.r.c).

S1 Jennings/Watts (Fn. So), $\$ 128, S_{4} 428$.

s2 Verdross/Simma, Volkerrecht $\left(198_{4}\right), \$$ sor.

33 Correia, I.C.J., Case Concerning East Timor, Public Sicting, 31st January 1995, CR 95/3, S. 58, Ausruge der mundlichen Verhandlung sind abgedruckt bei Krieger (Fn. 4), S. 377-398.

${ }_{4}$ Maffei, EJIL $_{4}$ (1993), S. $223,22.9$ (Fn. 20). 
Des weiteren hat Indonesien vorgebrachr, cs habe zur Unterstützung des Befreiungskampfes des abhängigen Kolonialvolkes von Ost-Timor gehandelt, da der Dekolonialisierungsprozeß in Ost-Timor durch bewaffnete Auseinandersetzungen gestört war. Aber auch diese Argumentation ist nicht geeignet, die indonesische Vorgehensweise zu rechtfertigen. Eine Rechtsüberzeugung, die militärische Hilfeleistungen für ein abhängiges Kolonialvolk als Ausnahme vom Gewaltverbot für zulässig erachtet, hat sich im Völkergewohnheitsrecht nicht herausbilden können." Zumindest solange die rechtmäßige Autorität in dem Gebiet den Prozeß der Sclbstbestimmung nach Treu und Glauben in dem Maße unterstützt, den die Umstände erlauben, müssen militärische Unterstützungshandlungen als rechtswidrige Einmischung in die inneren Angelegenheiten des Staates und als Verstoß gegen das Gewaltverbot betrachtet werden. ${ }^{56}$ Das Verfahren der Sclbstbestimmung war nach Portugals Angaben in Ost-Timor angemessen in Gang gesetzt. Die Entwicklungen Ende 1975 hätten nur vorübergehende Störungen des Prozesses dargestellt. ${ }^{57}$ Vertreter FRETILINs brachten zudem vor, daß die Intervention Indonesiens nicht gegen die Verwaltungsmacht Portugal, sondern gegen den von FRETILIN repräsentierten Großteil der Bevölkerung gerichtet war..$^{8}$ Damit war die Bitte um Unterstützung durch die pro-indonesischen Parteien nicht ausreichend, um die Intervention völkerrechtlich zu rechtfertigen. ${ }^{\text {i9 }}$

\section{b) Die "Inkorporation" als Bruch des Selbstbestimmungsrechts}

Da dic Generalversammlung Ost-Timor als Hoheitsgebiet ohne Selbstregierung nach Art. 73 SVN qualifiziert hat, ist das Volk von Ost-Timor Trägcr eines eigenen Selbstbestimmungsrechts. Indoncsien vertritt nun die Auffassung, daß das Volk von Ost-Timor bereits sein Selbstbestimmungsrecht endgültig ausgeübt habe, indem sich die Volksversammlung in Dili am 30. 5. 1976 für eine Vereinigung mit Indonesien ausgesprochen habe. Die Mitglieder der Volksversammlung seien durch ein Verfahren gewählt worden, das dem von Portugal vorgesehenen Verfahren des Gesetzes 7/75 entsprochen habe. Indonesien hebt hervor, daß die Übergangsregicrung 1976 mit Nachdruck darum bemüht gewesen sei, Repräsentanten der VN an dem Beitrittsverfahren teilhaben zu lassen, um alle erforderlichen Maßnahmen zu ergreifen, die der "Inkorporation "Wirksamkeit verleihen. Es sei allein Portugal und den es unterstützenden Staaten zuzuschreiben, daß alle Bemühungen in diese Richtung in den VN-Gremien blockiert worden seien. Die Volksversammlung sei stattessen unter Anwesenheit einiger Mitglieder des diplomatischen Korps und der internationalen Pressc zusammengetreten. Bevor das indonesische Parlament der Integration zugestimmt habe, habe es eine Beobachtermission nach Ost-Timor entsandt, die eine Mehrheit für eine Vereinigung mit Indonesien festgestellt haben will. ${ }^{60}$

Die Behauptung, das Volk von Ost-Timor habe sein Selbstbestinumungsrecht endgültig in einem rechtmäßigen Verfahren ausgeübt, hält einer rechtlichen Überprüfung nicht stand. Die Voraussetzungen der GVRes x S4 I (XV) vom I S. 12. 1960 sind nicht erfüllt. Diese Resolution ergänzt die grundlegende GVRes Is I4 (XV) vom 4. I 2. 1960 zur Dekolonialisierung. Nach Prinzip VI (c) des Annexes der Resolution

ss Cassese (Fn. 13), S. 199; Fischer (Fn. 47), \&57 Rdnr. 21.

s6 Jennings/Watts (Fn. so), $\$ 130$, S. 44 sf.

57 Correia (Fn. 53), 3oth January 1995, CR 95/2, S. 25.

58 Ramos Horta, SCOR, 3oth Year, 1864th Meeting, is. 12. 1975, para. 133

59 Maffei (Fn. 54), S. 229.

60 Statement made by H. E. President Suharto on 7 June 1976 in response to the adress presented by the delegation of the Provisional Government of East Timor, A/31/109, Annex V, S. 3 f. $=$ S/: 2097. 
I $4_{4}(\mathrm{XV})$ kann cin Hoheitsgebiet ohne Selbstverwaltung die Unabhängigkeit durch die Vereinigung mit einem anderen Land erlangen. Prinzip IX fordert hierfür, daß das Gebier einen fortgeschrittenen Stand an Selbstverwaltung mit cigenen unabhängigen politischen Institutionen erreicht hat. Die Integration soll das Ergebnis der freien Willensäußerung des Volkes sein, also eines demokratischen Prozesses, der unparteiisch geführt wird und auf einer allgemeinen Wahl beruht. Halten es die VN für erforderlich, so sollen sie den Prozeß überwachen können.

Das durch Kämpfe crschüttertc Ost-Timor hatte um die Jahreswende $1975 / 76$ kaum einen hinreichenden Grad an Selbstverwaltung erlangt. Die Situation hätte freie Wahlen unter VN-Überwachung verlangt. ${ }^{61}$ Die Bildung des Volksversammlung erfüllte nicht die von den VN aufgestellten Anforderungen. Sic kann nicht als unabhängige politische Institution betrachtet werden. Zum Zeitpunkt ihrer Errichtung kämpften dic indonesischen Truppen in Ost-Timor noch immer gegen die Kämpfer der FRETILIN und verursachten hohe Opfer in der Zivilbevölkerung. Angesichts der militärischen Besetzung des Landes konnte das Volk von Ost-Timor sein Selbstbestimmungsrecht nicht wirksam ausüben. Des weiteren war durch die Zusammensetzung die Allgemeinheit der Wahl nicht garantiert. ${ }^{62}$ Nur fünf der achtundzwanzig Vertreter wurden frei gewählt. Der Rest der Volksversammlung setzte sich aus zwei Repräsentanten von jedem der dreizehn Distriktsräte und aus zehn Häuptlingen und Führern der Stämme und religiösen Gruppen zusammen, die dic Übergangsregierung direkt bestimmte. ${ }^{63}$ Damit wich das Verfahren zum einen erheblich von demjenigen ab, das Portugal in dem Gesetz $7 / 75$ vorgesehen hattc. Zum andcren wurde die Wahl nicht durch unparteiische Beobachter überwacht. Der von Indonesien entsandten Mission kommt keine rechtliche Bedeutung zu, da im Zeitraum der Umfrage indonesisches Militär Ost-Timor besetzt hiclt. Repräsentanten der VN nahmen trotz der Einladungen der provisorischen Regierung nicht an der entscheidenden Sitzung der Volksversammlung teil. Zur Begründung bezog sich der Sicherheitsrat auf seine getroffenen Entscheidungen und damit - entegegen dem Vorbringen Indonesiens - auf rechtliche Gründe. Zu diesen Gründen zählte, daß der Sicherheitsrat mit der Angelegenheit beschäftigt blieb und daß der Sondergesandte des Generalsekretärs weiterhin unter seinem Mandat agierte. ${ }^{6} 4$ Hierin ist eine Weigerung des Sicherheitsrates zu sehen, die Anwesenheit Indonesiens in Ost-Timor anzucrkennen und eincn Dekolonialisierungsprozeß zu legitimieren, der außerhalb der VN-Mechanismen stattgefunden hat. ${ }^{65}$ Schließlich ist der indonesische Einwand hiergegen, dem Prinzip IX (b) der Resolution I 54I (XV) zufolge sei die Teilnahme der VN am Dekolonialisierungsprozeß nicht zwingend vorgeschrieben, nicht stichhaltig. Die Staatenpraxis und die Praxis der VN zeigen ein deutliches Muster für dic Durchführung eines Dekolonialisierungsprozesses, das freie Wahlen und Referenden unter VN-Überwachung vorsieht und das den Charakter eines Völkergewohnheitsrechtssatzes hat. ${ }^{66}$

Die Ereignisse der Jahre 1975 und 1976 können somit nicht als rechtmäßige Ausübung des Selbstbestimmungsrechts betrachtet werden. Vielmehr hat Indonesien mit der Integration Ost-Timors unter Anwescnheit seiner Truppen das Selbstbestimmungsrecht des Volkes von Ost-Timor in der Form des Rechts eines Kolonialvolkes auf Gründung eines eigenen Staates verlerzt.

61 Stellungnahme des Vorsızenden des VN-Dekolonialısıerungsausschusses, A/AC. $109 / 623,11,8,1980$, para. 9; Franck, AJIL 70 (1976), S.694, (699-701).

62 Guilhaudis, A.F.D.I. 23 (1977), S. 307 (314); Lawrence (Fn. 1), S. 95 .

$6{ }_{3}$ Act Number 1/A. D. 1976 of "Provisional Government of East Timor $\propto$ v. 2. 4. 1976, S/12 106, Appendix, S. sf.

64 Note des Präsıdenten des Sicherheitsrates v. 21.6.1976, S/12104.

65 Maffei (Fn. 54), S. 226 f.

66 Franck (Fn. 61), S. 701 ; Peters (Fn. I5), S. 57-67. 
Soweit ein Volk unter Fremdherrschaft auf Grund einer vorausgegangenen Annexion Trägcr des Selbstbestimmungsrechts ist, stellt die Aufrechterhaltung dieser rechtswidrigen Inkorporation eine weitere Verletzung des Selbstbestimmungsrechts durch Indonesien dar. ${ }^{67}$

\section{c) Vorliegen eines wirksamen, das Selbstbestimmungsrecht verdrängenden Gebietserwerbsgrundes?}

Hilfsweise behauptet Indonesien die Existenz eines wirksamen Erwerbstitels für die Inkorporation 1975. Eine Argumentationslinie bezieht sich auf die historische Verbindung zwischen Ost-Timor und Indoncsien in der Periode vor der Kolonialisierung. In dieser Zeit soll Ost-Timor Teil indonesischer Königreiche gewesen sein. Die Kolonialmächte hätten die Insel dann im 18. Jahrhundert willkürlich geteilt. Die Geltendmachung eines historischen Erwerbstitels beruht auf der Idee, daß wegen stark ausgebildeter historischer Vcrbindungen ein Gebiet, das von Kolonialmächten beherrscht wurde, dem vorkolonialen Souverän zurückzugeben sei, ungeachtet des Selbstbestimmungsrechts. ${ }^{68}$ Solche Argumente haben jedoch nur eine politische und keine rechtliche Grundlage. Zudem verlangte der IGH im Gutachten zur Westsahara für die Begründung eines historischen Rechtsanspruchs den Nachweis über die tatsächliche Ausübung von Staatsgewalt auf dem betreffenden Gebiet. Lehnsbeziehungen und religiöse Verbindungen reichen ihm nicht, einen Titel zu bcgründen und das Selbstbestimmungsrecht des dort lebenden Volkes zu verdrängen. ${ }^{69}$ Die vor allem auf ethnisch-kulturellen Argumenten beruhenden Beziehungen zu Indonesien sind daher nicht geeignet, einen wirksamen Gebictstitel darzulegen, der das Selbstbestimmungsrecht verdrängen könnte. Gerade die Rechtsprechung des IGH hat in dieseın Punkt zur Konkretisierung des Selbstbestimmungsrechts und zu seiner rechtlichen Anwendbarkeit beigetragen. ${ }^{7}$

Ein weiteres Argument zielt auf die Situation Ost-Timors als Kolonialenklave bzw. Mikrostaat. In Ost-Timor fehle es an einer ausgebauten Wirtschaft sowie an Rohstoffen, so daß es auf Grund der Rückständigkeit vorzuziehen sei, das Gebiet mit Indoncsien zu vereinigen. ${ }^{71}$ Hierbei kommen Erwägungen über die Sicherheit und Stabilität des Landes und der gesamten südostasiatischon Region zum Tragen. ${ }^{72}$ In Fällen der kolonialen Enklaven soll das Gebiec nicht als Selbstbestimmungseinheit behandelt werden, sondern in das Land integriert werden, von dem es wirtschaftlich und ethnisch völlig abhängig ist. In der Staatenpraxis ist dieses Konzept auf die Fälle der französischen und portugiesischen Niederlassungen in Indien angewendet worden. ${ }^{3}$ Daraus wurde in Teilen der Literatur die Schlußfolgerung gezogen, in OstTimor sei der Gedanke zur Anwendung gekommen, daß Interessen an Frieden und Sicherheit gegenüber dem abstrakten Selbstbestimmungsrecht vorrangig seien. ${ }^{7-4}$ Diese Rechrsfigur ist als Ausnahme vom Selbstbcstimmungsrecht jedoch nur im beschränkten Maße anwendbar. Bei größeren und eigenständigercn Gebieten, wie

\footnotetext{
67 Cassese (Fn. 13), S. 226 i.

68 Jennings, The Acquistion of Territory in Internationa! Law (1963), S. 76; Shaw, Internacional Law (1986), S. 265; derartige Anspruche sind von Marokko hezuglich der Westsahara und vom Irak bezuglich Kuwaits geltend gemacht worden.

69 Western Sahara, Advisory Opinion, I.C.J. Reports 1975, S. 12 (68 para. 162).

70 Bowring (Fn. I6), S. I65; Franck (I'n. 17), S. 369.

71 Martins, Vertreter von KOTA, SCOR, 3cth Ycar, 186, th Metring, 16. 12. 1975, paras.61-66.

72 Anwar Sani, Vertrecer Indonesicns, SCOR, jorh Year, 1864 th Meeting, 15. 12.1975 , para. 69.

73 Crawiord (Fn. 26), S. 384.

74 Engres, Netherlands International Law Review 24 (1977), S. 85 (89).
} 
Ost-Timor, ist der Gedanke nicht vertretbar. ${ }^{7 s}$ Für eine mögliche wirtschaftliche Eigenständigkeit Ost-Timors spricht, daß im Festlandsockel vor Ost-Timor Erdöl und Erdgasvorkommen vermutet werden. Schließlich behauptet Indoncsicn, Portugal habe das Gebiet derelinquiert. ${ }^{76}$ Der Zusammenbruch der portugiesischen Ordnung und der Rückzug Portugals 1975 sei der Grund für die indoncsische Invasion gewesen. Auch wenn Dereliktion ein Gebietsverlustgrund sein kann, ist der reine Rückzug aus dem Gebiet nicht ausreichend, eine Dereliktion zu implizieren. Erforderlich ist ein Handeln mit dem entsprechenden animus derelinquendi. Der Wille kann zwar aus der fehlenden Ausübung von Staatsgewalt über ein Gebiet für einen längeren Zeitraum hergelcitet werden. Proteste des betroffenen Staates sind aber zu berücksichtigen. Eine nur vorübergehende Schwächung der tatsächlichen Ausübung der Hoheitsgewalt ist solange nicht ausreichend, einen Verlust der Hoheitsgewalt zu begründen, solange eine Vermutung besteht, daß der Staat den Willen hat, die Hoheitsgewalt wiederzuerlangen. ${ }^{77}$

Portugal bestreitet, mit animus derelinquendi die Insel verlassen zu haben. Der Rückzug auf die Insel Atauro sei norwendig gewesen, da das portugiesische Militär nicht in der Lage gewesen sei, den Bürgerkriegsparteien zu widerstehen. ${ }^{73}$ Angesichts der portugiesischen Bemühungen um eine Konfliktlösung im Sommer x975 kann nur von einem vorübergehenden Verlust der Kontrolle über das Gebiet gesprochen werden. Erst die indonesische Invasion führte dazu, daß Portugal endgültig aus Ost-Timor verdrängt wurde. Portugal hat seitdem gegen die »Inkorporation " protestiert. ${ }^{79}$ Unmittelbar nach der Invasion brach es die diplomatischen Beziehungen zu Indonesien ab. ${ }^{*}$ Gegenwärtig kommt Portugal seinen Verpflichtungen als Vcrwaltungsmacht auf Grundlage seiner Verfassung nach und übt seine Funktion als Verwaltungsmacht weiterhin vor den VN-Gremien aus. ${ }^{{ }_{1}}$

Indonesien kann keine wirksamen Ansprüche auf Ost-Timor begründen. Wegen der Verletzung des Selbstbestimmungsrechts ist die „Inkorporation « unwirksam. Auf Grund des Vcrstoßes gegen das allgemeine Gewaltverbot als ciner Norm des zwingenden Völkerrechts ist der unter Gewaltanwendung vorgenommene Gebietscrwerb Ost-Timors durch Indonesien völkerrechtlich unwirksam. ${ }^{82}$ Daher besteht der Status Ost-Timors als portugiesisches Hoheitsgebiet ohne Selbstregierung fort.

75 Elliot, ICLQ 27 (1978), S. 238 (248); Simpson, Hastings International and Comparative Law Review i7 (1994), S. $323(342)$.

76 Stellungnahme von Nasier, Vertreter Indonesiens, A/AC. 109/PV. 1406, 28.7. 1992, S. 31; siehe auch Australiens Vorbringen vor dem IGH: Griffiths I.C.J., Case Concerning East Timor, Public Situng, 6th February 1995, CR 95/7, S. 43-44; Burmester, (ebd.), 8th February 1995, CR 95/9, S. 8-10.

77 De Zayas in: Bernharde (ed.), EPIL, Instalment to (1987), S. $494 \mathrm{f}$.

78 Stellungnahme von Quartin-Santos, Vertreter Portugals, A/AC. 109/PV. 1406, 28. 7. 1992, S. 39 f.; Correia $\left(\mathrm{Fn}_{\mathrm{n}}, 53\right), \mathrm{S} .57 / 59$.

79 Z. B. Stellungnahme der portuglesischen Regierung, in: Commission on Human Rights (Fn, 12), Annex II.

80 Kommuniqué des portugiesıschen Mınisterrates v. 7.12,1975, S/11899, Annex.

81 Art. 293 der portugiesischen Verfassung von 7.7.1989: zur Weitergabe von Informationen nach Art. 73 (e) SVN: A/so/214, 9.6.1995; Cassese in: Lowe/Fitzmaurice (Hrsg.), Fifty Years of the International Court of Justice (1996), S. 35 I (36r) behaupret auf Grundlage des Westsahara-Gutachtens den Rechtssatz, daß die Dereliktion durch dic alte Kolonialmacht das Gebiet nicht zu terra nullus mache.

82 Dorr (Fn. 44), S. 127. 
Im folgenden nuß das Augenmerk auf die Praxis der Staatengemeinschaft gerichtet werden. Nachdem festgestellt werden konnte, daß die Anrvendung des Selbstbestimmungsrechts in seinen verschiedenen Ausprägungen zu einer klaren rechrlichen Beurteilung führt, bleibt die Frage, ob die mangelnde Bewältigung des Konflikts auf die fehlende politische Durchsetzbarkeit des Anspruchs zurückzuführen ist. Zur Problematik der politischen Durchsetzbarkeit tritt ein weiteres rechtliches Argument hinzu. Das Selbstbestimmungsrecht ist in der Rechtsprechung des IGH als eine wesentiche Norm des geltenden Völkerrechts mit einer Wirkung erga omnes anerkannt. Trotzdem ist zweifelhaft, ob auf Grund eines Verstoßes gegen das Selbstbestimmungsrecht eine selbständige Pflicht der Drittstaaten besteht, die "Inkorporation " nicht anzucrkennen. ${ }^{83}$ Daher stellt sich die Frage, inwieweit die VN-Resolutionen den Staaten eine Pflicht zur Nichtanerkennung auferlegen. Zur Ermittlung ist neben dem Wortlaut der Resolutionen auch die Staatenpraxis heranzuziehen.

Zudem könnte dic Praxis der Staaten unter Bcrücksichtigung des Zeitablaufs Auswrirkungen auf den Status des Gebietes zeitigen. ${ }^{{ }^{4}}$ Auch wenn die Annexion als solche keinen Titel mehr darstellt, ist davon die Frage zu unterscheiden, ob ein weiterer eigenständiger Rechtsgrund hinzutreten kann. Ein solcher Rechtsgrund könnte cine mit Gcwalt geschaffene tatsächliche Lage, dic zu einem vcränderten Sachverhalt geführt hat, in einen wirksamen Gebietserwerb umwandeln. Ob dies angesichts des zwingenden Gewaltverbotes und des Selbstbestimmungsrechts eines Volkes überhaupt zulässig ist, ist umstritten." Bejaht man die Möglichkeit z. B. der Ersitzung oder eines Titels aufgrund historischer Konsolidierung, kommt der $\mathrm{Hal}$ tung der Drittstaaten eine wesentliche Bedeutung für die Frage zu, ob ein solcher Titel wirksarn begründet worden ist. ${ }^{86}$

\section{a) Ost-Timor vor den VN}

Der Sicherheitsrat hat auf die »Inkorporation $*$ Ost-Timors mit der Verabschiedung der Resolution 384 (1975) vom 22. I 2. 1975 und 389 (1976) vom 22.4. 1976 reagiert. In beiden Resolutionen wird hervorgehoben, daß das Volk von Ost-Timor Träger des Selbstbestimmungsrechts ist. Ebenso ruft der Rat die Staaten auf, die territoriale Integrität Ost-Timors zu respektieren, und fordert die Regierung Indoncsiens zum Rückzug ihrer Truppen aus Ost-Timor auf. Auclı die Gencralversammlung hat sich mit dem Fall Ost-Timor beschäftigt. In der Resolution 3485 (XXX) vom 1 2. 1 2. 1975 wird, anders als in den Sicherheitsratsresolutionen, auf Art. 2 Abs. 4 SVN verwiesen. Nach dem Vollzug der »Inkorporation" stellt die Resolution 32/34 vom 28. I r. 1977 deren Unwirksamkeit fest, da die Bevölkerung des Gebietes ihr Selbstbcstimmungsrecht nicht frei ausüben konnte.

83 Vgl. Gowlland-Debbas, Collective Responses to Illegal Acts in Intcrnational Law (1990), S. 287 f.; die Staatenpraxis behandelt die Annexion Ost-Tımors in erster Linie als Verstoß gegen das Selbstbestimmungsrecht und nicht als Verstoß gegen das Gewalt- und das Annexionsverbot, ber denen das Bestehen einer selbstandigen Nichtanerkennungspflicht angenommen wird, Dörr (Fn. 44), S. I 20 f

84 Fonteyne, Austr. Journal of Intern'l Affairs 45 (1991), S. 170 (178); Hilpold (Fn. 16), S. 78 ; ablebnend: Chinkin, EJIL 4 (1993), S. 206 (212); Judge Skubiszewski, dissenting opinion, East Timor, Judgment, I.C.J. Reports 1995, S. 224 (26I f. paras. 1 22-133).

8 s Siehe zum Streitstand z. B. Zimmer, Gewaltsame territoriale Veranderungen und ihre volkerrechtliche Legitimation (1971), S. 18-82; ablehnend Gloria in: Ipsen (Fn. 47), \$23 Rdnr. 63; gegen eine Konsolidierung bei bestehendem Anspruch auf Selbstbestimmung Hilpold (Fn. 16), S. 72 f., mit Einschrankungen auf S. 78 .

86 Jennings/Watts (Fn. 50), $\$ 275$, S. 705 f./71s f.; Seidl-Hohenveldern, Volkerrecht (1994), Rdnr. 1157; de Visscher, Théoriés et Réalıtés en Droit International Public (1970), S. $226 \mathrm{f}$. 
Die Resolutionen legen keine ausdrücklichc Verpflichtung zur Nichtanerkennung auf. ${ }^{87}$ Auch wenn beide VN-Organe darin übereinstimmen, daß kein wirksamer Akt der Selbstbestimmung stattgefunden hat, ${ }^{88}$ ist den Resolutionen kcine aus der Verletzung des Selbstbestimmungsrechts resultierende Pflicht zu entnehmen, jegliche Gebictshoheit Indonesiens für Ost-Timor nicht anzuerkennen und demgemäß nur mit Portugal über das Gebiet zu verhandeln. ${ }^{89}$ Dies ergibt sich daraus, daß dic Sicherheitsratsresolutionen zum einen nicht verbindlich nach Kapitel VII SVN ergangen sind und zum anderen nicht in ciner verbindlichen Sprache verfaßt sind, die eine Verpflichtung aus Art. 2s SVN zur Folge hätte. Sanktionen sind nicht empfohlen oder gar angeordnet worden..$^{\circ}$ Begriffe wie Invasion oder Aggression wcrden nicht benutzt, um die indonesische Intervention zu charakterisieren. Die "Inkorporation « wird bis auf einige allgemein gehaltene Verweise auf die territoriale Integrität der Staaten nicht unter dem Gesichtspunkt des Annexionsverbotes betrachtet. Der Sichcrhcitsrat schien nicht bereit zu sein, Indonesien wegen der Verletzung des Gewaltverbotes zu verurteilen.

Die Rechtswidrigkeit der indonesischen Intervention und Annexion spiegelt sich auch nur teilweise in der weiteren Entwicklung vor den VN wider. Der Sicherheitsrat hat sich seit 1976 nicht mchr mit der Situation in Ost-Timor beschäftigt. Zwischen $197 \mathrm{~S}$ und 1982 hat die Generalversammlung regelmäßig Resolutionen über Ost-Timor verabschiedet, jedoch mit schwindenden Mehrheiten. ${ }^{9}$ Seither hat die Generalversammlung kcine Resolutionen mehr über Ost-Timor verabschiedet. Es bestand die Gefahr, daß dic erforderliche Mehrheit nicht mchr zustandegckommen wäre. ${ }^{92}$ Die Ziclrichtung der Resolutionen änderte sich mit der Resolution 34/40 vom 21. I I. I 979. In ihr entfallen die Verweise auf das Gewaltverbot des Art. 2 Abs. 4 SVN und auf Art. I I Abs. 3 SVN, der vorsieht, daß die Generalversammlung die Aufmerksamkeit des Sicherheitsrates auf Situationen lenken kann, die geeignet sind, den Weltfrieden zu gefährden. Auch der Verweis auf die territoriale Integrität Ost-Timors entfällt. Weder in der Präambel noch im operativen Teil der Resolution wird entgegen der üblichen VNPraxis auf die früher verabschiedeten Resolutionen Bezug gcnommen. Die Resolution konzentriert sich auf die humanitäre Situation als Folge der Hungerkatastrophe in Ost-Timor. Die Ausübung des Selbstbestimmungsrechts im Rahmen der VN bleibt aber als Forderung in den Resolutionen cnthalten. Zwar erwähnt die letzte verabschiedete Resolution 37/30 vom 23. 11 . 1982 im operativen Teil nicht, daß das Volk von OstTimor Träger diescs Rechts ist, sie bezieht sich aber wieder auf die früheren Resolutionen. In der Entwicklung mag man Anzeichen dafür sehen, daß die Generalversammlung die Inkorporation als fait accompli betrachtet. ${ }^{33}$

87 Die Praxis, Mitgliedstaaten eine Verpflıchtung zur Nichtanerkennung aufzuerlegen, hat der Sicherheitsrat in den Fällen Sudrhodesiens S/Res/217 (1965), 277 (1970), 288 (1970), 328 (1973), 423 (1978), 445 (1979), 448 (1979), der sudafrikanischen bomelands 402 (1976); des israelischen Sechs-Tage-Krieges 242 (1967); der Ausrufung der Turkischen Republik Nordzypern $4_{4} 1$ (1983) und der irakischen Invasion in Kuwait 662 (1990) verfolgt.

88 Die Mehrheit der Staaten brachte in den Debatten im Dezember 1975 und im April 1976 (SCOR, 30th Year, 1864 th-1869th Meeting und 3 ist Year, I90.ith ig I sth Meeting) zum Ausdruck, daß das Selbscbestimmungsrecht der Volker verletzt worden set. Indonesitris Position wurde allcin von einigen arabischen und asıatischen Staaten unterstütz.t, z. B. Stellungnahme des Vertretcrs Malaysias, SCOR, 3 Ist Year, ro1 th Meeting, paras. $22-30$.

89 East Timor, Judgment, 1.C.J. Reports 1995 , S. 90 ( 103 para. 31 ).

90 Im Fall des irakischen Einmarsches in Kuwait im August 1990 verhangte der Sicherheirsrat z. B. mit der Resolution 661 (1990) wirtscbaftliche Zwangsnaßnabmen nach Kapitel VII SVN

$91 \mathrm{~A} / \mathrm{Res} / 3485(\mathrm{XXX})$ angen, am 12. 12.1975 mit 72 7.u 10 Summen bei 43 Enthaltungen; $31 / 53$ angen. am 1. 12. 1976 mit 68:20:49; 32/34 angen. am 28. 11. 1977 mit 67:26:47; 33/39 angen. am 13.12. 1978 mit 59:31:44; 34/40 angen. am 2 I. II. 1979 mit 62:31:45; 35/27 angen. am r1. Ir. 1980 mit $58: 35: 46 ; 36 / 50$ angen. am 24. 11, 1981 mit $54: 42: 46$ und $37 / 30$ angen. am $23.11,1982$ mit 50:46:50.

92 Chinkin (Fn. 84), S. 212 Fn. 29

93 Cassese (Fn. 13), S. 223. 
Dennoch darf bei der Frage, ob ein rechtmäßiger Anspruch von der Staatengemeinschaft aufrechterhalten wird, nicht allein auf die Hauptorgane der VN zurückgegriffen werden. Die Generalversammlung fordert 1982 in ihrer letzten Resolution 37/30 zu Ost-Timor den Dekolonialisierungsausschuß auf, die Situation weiterhin als Problem der Dekolonialisierung zu betrachten. Die VN erkennen Portugal weiterhin als Verwaltungsmacht des Territoriums an. Ost-Timor wird weiterhin in der Liste der Hoheitsgebiete ohne Sclbstregierung nach Kap. XI, Art. 73 SVN geführt. ${ }^{94}$ Seit 1977 hat die portugiesische Regierung in ihrer Funktion als Verwaltungsmache OstTimors zwar jährlich den Generalsekretär informiert, daß sie auf Grund der Anwesenheit der indonesischen Streitkräfte nicht in der Lage war, ihrer Bcrichtspflicht nach Art. 73 c SVN nachzukommen. 951994 aber wies Portugal in Ausübung dieser Pflicht auf Umstände hin, die einer von Indonesien behaupteten Verbesserung der Situation in dem Gebiet entgegenstehen. Portugal hat in den Gremien der VN seinen Protest gegen die Invasion Indonesiens aufrechterhalten, indem es auf die Rechtswidrigkeit der "Inkorporation « durch Indonesien und auf die Verstöße gegen Menschenrechte hingewicsen hat. ${ }^{6}$ Durch die Resolution $37 / 30$ wurdc auch der Generalsekretär beauftragt, Gute Dienste zur Vermittlung zwischen den Parteien zu leisten. Unter seiner Schirmherrschaft wird seitdem der Dialog zwischen den Konfliktpartcien fortgesetzt. Über die Aktivitäten bcrichtct der Generalsekretär an die Generalversammlung. ${ }^{97}$ Dcr Arbeit des Dekolonialisierungsausschusses und vor allem den Vermittlungsbemühungen des Generalsekretärs kommt gegenwärtig die Schlüsselfunktion dafür zu, die legitimen Bestrebungen des Volkes von Ost-Timor nach Selbstbestimmung zu wahren..$^{8}$

\section{b) Haltung der Drittstaaten}

Dic Reaktioncn der Drittstaaten können in drei Gruppen unterteilt werden. Einige Staaten vertreten den Standpunkt, daß Ost-Timor rechtmäßig in Indonesien eingegliedert worden sei. Sie haben eine de iure Anerkennung der "Inkorporation" ausgesprochen. $\mathrm{Zu}$ diesen Staaten gehören u. a. Bangladesh, Indicn, Iran, Irak, Jordanien, Malaysia, Marokko, Oman, die Philippinen, Saudi Arabien, Singapur, Surinam und Thailand. ${ }^{99}$ Andere Staaten vertreten die Ansicht, daß dem Volk von Ost-Timor weiterhin cin Selbstbestimmungsrecht zukomme, verurteilen die Art und Weise der "Inkorporation «, aber akzeptieren ausdrücklich oder stillschweigend die indonesische Souveränität über Ost-Timor als Tatsache. Zu diesen Staaten gchören Kanada, Japan, Mauretanien, Neusceland, Papua Neuguinea, Schweden und die USA. Ihre Haltung kann als de facto Anerkennung gewertet werden. In den Fällen Kanadas, Neuseelands und der USA wird auch von einer de iure Anerkennung gesprochen. ${ }^{100}$ Eine dritte Gruppe crkennt die "Inkorporation « weder de iure noch

94 East Timor, Workıng Paper Prepared by the Secretariat, A/AC. 109/1187, 7. 7.1994, S. 2 para. 3

95 Z.B. $\mathrm{A} / 35 / 233$ und $\mathrm{A} / 48 / 130$.

$96 \mathrm{~A} / 49 / 184 ;$ Quartın-Santos (Fn. 78), S. 26

97 Z.B. A/35/524 und $\mathrm{A} / 50 / 436$.

98 Anlaßlich des achten Treffens zwischen Indonesien und Portugal im Juni 1996 in Genf erklarte der indonesische Außenminuster seine Bereitschaft, die Frage Ost-Timors wieder yor den VN zu erortern; The Jakarta Post, 13.6.1996.

99 Z.B. Stellungnahme des Vertreters Singapurs im 4. Komitee $\operatorname{der}$ GV, A/C.4/37/SR.13, is. 1 1. 1982 , para. 2 2.

100 Kanada: Secretary of State for External Affarrs, House of Commons Debates, 10.9.1991, S. 2310, abgedr. in: CYIL 30 (1992), S. 366 ; USA: Statement before the Subcommutee on Asien and Pacific Affairs of the House Foreign Affairs Committee on 14.9.1982, Ambassador Holdridge, abgedr. in: Department of Statc Bulletin 82 (1982), S. 29; so interpretiert von Großbritannien: Reply to an oral question of Minister of State, Foreign and Commonwealth Office, HL Debs,, vol. 532, col. 1146:25.11. 1991, abgedr. in: BYLL 62 (1991), S. 569 . 
de facto an. Die Mitgliedstaaten der EU und der WEU gehören zu dieser Gruppe. ${ }^{101}$ Auch die Bundesrepublik Deutschland hat die "Inkorporation« Ost-Timors nicht anerkannt. ${ }^{102}$ Im Juni 1996 hat der Rat der Europäischen Union einen Gemeinsamen Standpunkt zu Ost-Timor festgelegt, in dem er unter Bczug auf die Aktivitäten der VN eine Regelung der Frage im Weg des Dialogs sowie die Einhaltung der Menschenrechte in dem Gebiet als Zielsetzung nennt. ${ }^{103}$

Staaten der zweiten und dritten Gruppe haben jedoch Verträge mit Indonesien abgeschlossen, z. B. Doppelbesteuerungsabkommen, in denen das Gebiet Indonesiens in Übereinstimmung mit seinen eigenen Gesetzen definiert wird. ${ }^{104} \mathrm{Z}$ war besteht im Völkerrecht der Grundsatz, daß die Ausdehnung der territorialen Anwendbarkeit von Verträgen auf alle Gebiete, über die die Vertragsstaaten Gebietshoheit ausüben, keine Anerkennung der rechtmäßigen Ausübung der Gebietshoheit bedeutct. ${ }^{105}$ Seit der "Inkorporation « bedeutet aber jede Bezugnahme auf das Gebiet als Tcil Indonesiens eine Anerkennung der indonesischen Befugnis, das Gebiet völkerrechtlich zu vertreten. ${ }^{106}$ Seit 1976 ist kein Vertrag mit Indonesien abgeschlossen worden, der einen Vorbehalt oder eine Definition enthiclte, in der das Gebiet Ost-Timors aus dem tcrritorialen Anwendungsbereich des Vertrages ausgeschlossen würde. Zwischen 1976 und 1992 hat Indonesien in diescr Form Doppelbesteuerungsabkommen mit $3 \mathrm{I}$ Staaten abgeschlossen und ist Vertragspartei von 4 $\mathrm{r}$ multilateralen Verträgen geworden. Selbst Portugal hat dagegen nicht protestiert. ${ }^{107}$ Hierin kann ein Indiz dafür gesehen werden, daß die Staaten den status quo Ost-Timors anerkennen und nicht annehmen, daß die VN-Resolutionen Drittstaaten Verpflichtungen auferlegen, keine Verträge mit Indonesien abzuschließen, die das Gebiet Ost-Timors betreffen.

Auf diese Weise trägr die Staatenpraxis zu einer Konsolidierung der indonesischen Position bei, auch wenn Indonesien vor allem wegen der Haltung der VN bis heute keinen wirksamen Titcl auf Ost-Timor begründen kann. Die Ancrkennung der indonesischen Souveränität über das Gebiet einerseits und die Unterstützung des Selbstbcstimmungsrechts des Volkes von Ost-Timor andererseits geraten aber in cinen Widerspruch zueinander. Die Anerkennung friert den status quo ein, während dic Selbstbestimmung aufgeschoben wird. ${ }^{\text {108 }}$

\section{Ost-Timor vor dem Internationalen Gerichtshof}

Auch der Umstand, daß der Internationale Gerichtshof in Den Haag 1995 mit dem Status Ost-Timors befaßt war, hat die Frage nicht klären können, welche Verpflichtungen Drittstaaten obliegen, wenn das Selbstbestimmungsrecht verlctzt wird. Ebensowenig ist die indonesische Verhaltensweise gerichtlich beurteilt worden.

101 Mınister of State, FCO, HC D.bs., vol. $\{2$, col. 901 : 25. 1. 1984, abgedr. in: BYIL S5 (1984), S. 431; Unterrichtung durch die deutsche Delegation in der Versammlung der WEU uber die Tagung der Versammlung v. 2. bis 5. 12.1991 in Paris, Entschließung 84, BT-Drs. $12 / 2202,9.3 .1992$, S. 4 If.

102 Antwort der Bundesregierung auf die Kleine Anfrage des Abgeordneten Verheyen und der Fraktion DIE GRÜNEN, BT-Drs. $10 / 1246,5 \cdot 4 \cdot 1984$, S. 3 .

103 Gemeinsamer Standpunkt v. 25.6. 1996 betreffend Ost-Tımor, vom Rat aufgrund von Artikel J. 2 des Vertrages uber dic Europaische Union festgelegt, EG-Amtsblatt Nr. L 168/2.

${ }_{10}$ Übersicht hierzu beı Krieger (Fn. 4 ), S. 291-297.

105 Dochring, ZaoRV $27(1967), \mathrm{S}_{4} 4_{3}(488)$.

106 Pellet I.C.J., Case Concerning East Timor Public Sitting, 7th February 1995, CR 95/8, S. 22; Bowett (ebd.), 9th February 1995 , CR 95/10, S. $38 \mathrm{f}$.

107 Argentınıen z. B. hat 1990 aus Anlaß seines Beıtritts zum Übereınkommen zum Schutz der Ozonschicht einen Vorbehalt bezugheh der Anwendung des Abkommens auf die Falkland-Inseln als britischem Hoheitsgebiet abgegeben, BYIL 6I (1990), S. 55s.

108 Judge Skubiszewsku (Fn. 84), S. 268 para. 141. 
Australien und Indonesien schlossen 1971 und 1972 Verträge über die gemeinsamen Seegrenzen ab. Das Gebiet zwischen Australien und Ost-Timor war davon nicht erfaßt. Verhandlungen mit Portugal blieben erfolglos. ${ }^{109}$ Mit der Aufnahme der Verhandlungen über die Seegrenzen am I 4. 2. 1979 erkannte Australien die "Inkorporation « de iure an, wobei es die Art und Weise der "Inkorporation « in den Erklärungen bedauerte. ${ }^{10} 1989$ schlossen Indonesien und Australien einen Vertrag über die gemeinsamc Ausbeutung des zwischen Ost-Timor und der australischen Küste licgenden Festlandsockels ab." In der Präambel und in Art. 2 des Vertrages wird Ost-Timor als Provinz Indonesiens bezeichnet." Portugal hat gegen dic Verhandlungen unter Verweis auf das Selbstbestimmungsrecht des Volkes von Ost-Timor und seine eigenen Rechte als Verwaltungsmacht protestiert und schließlich eine Klage vor dem IGH anhängig gemacht."'s

\section{b) Indonesien als notwendige dritte Streitpartei}

Portugal behauptete die völkerrechtliche Verantwortlichkeit Australiens für den Abschluß und die Umsctzung eines Vertrages mit Indonesien, der die Erforschung und Ausbeutung des Festlandsockels zwischen Ost-Timor und Australien zum Gegenstand hat, sowic für weitere Verhandlungen über die Bestimmung der Seegrenzen in diesem Gebiet. Mit einer Mehrheit von ${ }_{4} 4$ zu 2 Stimmen hat der Gerichtshof die portugicsische Klagc mangels Jurisdiktion abgewiesen. ${ }^{1+}$ Materielle Rcchtmäßigkeitsfragen hat der IGH nicht erörtert.

Der IGH kann kein Urteil über die Rechtmäßigkeit des Verhaltens eines Drittstaates fällen, wenn dieser Staat nicht seine nach Art. 36 Abs. I IGH-Statur erforderliche Zustimmung zur Jurisdiktion des Gerichtshofs abgegeben hat. Indonesien selbst hat sich der Gerichtsbarkeit des IGH nicht unterworfen. Jeder Beurteilung des Verhaltens Australiens hätte aber cinc Untersuchung der Rechrmäßigkeit der indoncsischen Vorgchensweise als eigentlichem Kern des Streites vorausgehen müssen. Das sogenannte Monetary Gold-Prinzip gelangte zur Anwendung. Auch wenn das Sclbstbestimmungsrecht als eine wesentliche Norm des geltenden Völkerrechts mit Wirkung erga omnes anerkannt ist, muß die Geltung dieses Rechtes von der Frage der Jurisdiktion des IGH unterschieden werden." is

Zwar ließ der IGH den Fall an der fehlenden Gerichtsbarkeit scheitern, aber er traf dennoch einige bedeutende materiellrechtliche Feststellungen: Erstens bestätigte er,

109 1973 Austl. T.S. No. 31 und 1973 Austl. T. S. No. 32; vgl. Martin/Pickersgill, Harvard Intn'l L. J. 32 (1991), S. 566.

110 *The negotiations when they start, will sıgnify de iure recognition by Australia of the Indonesıan incorporation of East Timor.a Announcement made by Minister of Foreign Affairs, 15. 12. 1978, abgedr. in: Australian Year Book Intn'l Law 8 (1978-80), S. 28 I.

11 Abgedr. in: ILM 29 (1990), S. 475-537; Australien setzte den Vertrag 1990 um: Australian Petroleum (Australia-Indonesia Zone of Cooperation) Act 1990, No. 36 of 1990; siehe zur innerstaatlichen Überprufung der Rechtmaßigkett des Vertrages in Australien: Fitzgerald, ICLQ 44 (1995), S. 643-649.

112 In den oben genannten Doppelbesteuerungsabkommen dagegen wird das Gebiet Indonesiens nur in Übereinsummung mit seınen eigenen Gesetzen definıert.

113 I.C.J., Case Concernıng East Timor, Application Instituting Proceedings of the Government the Portuguese Republic, filed in the Registry of the Court, 22.2.1991; abgedr. ber: Krieger (Fn.4), S. $371-376$.

114 East Timor, Judgment, I.C.J. Reports 1995 , S. 90 (106 para. 38 ).

I s East Timor, Judgment, I.C.J. Reports 1995, S. 90 (102 paras. 28f.); das Prunzip ist erstmals im Fall Monctary Gold Removed from Rome in 1943, I.C.J. Reports 1954, S. 32 angewendet worden; siche hierzu Cbinkin, ICLQ 45 (i996), S. 7 I 2 (7171.); Jouannet, RGDIP 100 (1996), S. 673 ff.; Zimmermann, ZaoRV ss (1995), S. rosiff. 
daß das Volk von Ost-Timor Träger des Selbstbestimmungsrechts ist. Zweitens stellte der IGH fest, daß die Resolutionen keine Nichtanerkennungspflicht für die Mitgliedstaaten der VN begründen. Fragen des Verstoßes gegen das Gewaltverbot wurden nicht erörtert, ${ }^{16}$ weil Portugal sein Klagevorbringen auf die Frage des Selbstbestimmungsrechts des Volkes von Ost-Timor, die Pflichten von Drittstaaten einem Träger des Selbstbestimmungsrechrs gegenüber und auf Portugals Status als Verwaltungsmacht beschränkt hatte. Drittens hob der IGH hervor, daß aus dem Festhalten an dem Status Portugals als Verwaltungsmacht des Gebietes nicht die Schlußfolgerung gezogen werden könne, daß Drittstaaten nur mit Portugal Verträge über das Gebiet abschließen dürften. "7 Schließlich betonte der Gcrichtshof mehrmals, daß weiterhin für die beiden Streitparteien das Volk von Ost..'1imor Träger des Selbstbestimmungsrechts ist und das Gebiet trotz der de iure Anerkennung auch für Australien ein Hoheitsgebiet ohne Selbstregierung bleibt. ${ }^{118}$

Zwar mag es unbefriedigend erscheinen, daß der IGH Indonesiens Verhalten auf Grund des Monetary Gold-Prinzips nicht rechtlich überprüft hat, aber es handelt sich dabei um eine notwendige Konsequenz des Gefüges der internationalen Gerichtsbarkeit, die weder zwingend noch umfassend ist.

\section{Fortbestand als portugiesisches Hobeitsgebiet obne Selbstregierung}

Die Untersuchung statusrechtlicher Fragen hat ergeben, daß Ost-Timor den völkerrechtlichen Status eines portugiesischen Hoheitsgebietes ohne Selbstregierung im Sinn des Kapitel XI SVN beibehält. Der Dekolonialisierungsprozeß ist noch nicht beendet. Das Volk von Ost-Timor hat das Recht auf freie Selbstbestimmung. Die Pläne der einzelnen politischen Gruppierungen sehen eine langfristige Lösung des Konflikts in der Zuerkennung eines höheren Grades an Autonomie durch Indonesien vor, der schließlich zu einem Referendum über die Unabhängigkeit unter der Überwachung der VN führen soll.' "9 Das Beispiel Eritreas zeigt dabei, daß durch ein international unterstütztes Referendum cine friedliche Konfliktlösung erzielt werden kann. Jedoch tritt hier wieder ein wesentliches Problem der Anwendbarkeit des Selbstbestimmungsrechts zutage: die Frage nach der Ermittlung des Rechtsträgers. Bevor ein Referendum organisiert werden kann, muß klar sein, wer zur Gruppe der Berechtigten, wer zum Volk gehört. ${ }^{10}$ Die Umsiedlungsstrategie und die von Menschenrechtsorganisationen verurteilte Bevölkerungspolitik Indonesiens gefährdet die eigene Identität des Volkes und trägt erheblich dazu bei, das historische Unrecht zu konsolidieren. ${ }^{121}$

116 Zur Frage der bestchenden Nichtanerkennungspflicht auf Grund des Verstoßes gegen das Gewaltverbot: Judge Skuhiszcwski (Fn. 84), S. 261 f. paras. 122-133.

117 East Timor, Judgmcut, I.C.J. Reports 1995, S. 90 (103 f. paras. 3 If.)

I1 8 East Timor, Judgment, I.C.J. Reports 1995, S. 90 ( 105 para. 37); zur rechtlichen Widerspruichlichkeit der australischen Positionen: Judge Weeramantry, dissenting opinion, East Timor, Judgement, I.C.J. Reports 1995, S. 139 (201 f.); Judge Skubiszewski (Fn. 84), S. 2601. paras. 117-121.

1 Ig Punkt s des Offenen Briefes des Msg. Belo v. Juli 1994: Ramos Horta, East Timor: The Struggle for Sclf-Determination and its Future in Southeast Asia, Ansprache vor dem Royal Institute of International Aftairs, Chatham House, I.undon 23.4. 1996.

120 Trotz grundsatzlicher Finigung bestehen zur Zeit z. B. keine konkreten Aussicbten, daß eın Referendum unter Überwachung der VN in der Westsahara tatsachlich durchgefuhrt wird, da einc Einigung über die Abstimmungsberechtigten nicht erreicht werden kann; S/Res/1056 v. 26. 5. 1996; sowie A/Res/s1/143 v. 10. 2.1997.

121 Stellungnahme der East Timor Alert Network, GAOR, Special Committec, 1435 th Meeting, 13. 7.1994 . A/AC. $109 /$ PV. 1435, S. 79. 
Das Selbstbestimmungsrecht der Völker ist trotz aller juristischer Auslegungsprobleme gerade im Dekolonialisierungskontext hinreichend klar definiert, um auch bei der Anwendung im Einzelfall zu rechtlich begründeten Lösungen zu führen. Dennoch wird jede Partei bemüht sein, die rechtliche Beurteilung ihres Handelns durch Dritte mit ihren eigenen Argumenten zu beeinflussen. Damit wird sic aber nur erfolgreich sein, wenn sie sich im Rahmen des rechtlich Vertretbaren hält. Denn selbst in Fällen, in denen die Anwendung einer rechrlichen Regel nicht zu cinem eindeurigen Ergebnis führt, ist jedenfalls eine Grenzziehung zwischen vertretbaren und nicht mehr vertretbaren Entschcidungen möglich. ${ }^{122}$ Damit liegt das Problem aber nicht in der Anwendbarkeit der Rechtsnorm, sondern in der Struktur des Völkcrrechts, das als dezentrales Recht selten letztverbindliche Entscheidungen zentraler Organe erlaubt, die zwangsweise durchsetzbar wären. ${ }^{23}$ Der Beurteilungsspielraum und die Vielfalt der die rechtliche Beurteilung vornehmenden Aktcure führen zu Durchsetzbarkeitsproblemen des Anspruchs, die durch den fehlenden politischen Willen sowohl der betroffenen Staaten selbst als auch dritter Staaten und der internationalen Organisationen verstärkt werden. ${ }^{124}$ Ein besondercs Instrument, dic Durchsetzung völkerrechtlicher Normen zu gewährleisten, ist die Nichtanerkennungspflicht. ${ }^{125}$ Daß dic Vcrletzung des Selbstbestimmungsrechts keine völkergewohnheitsrechtliche Nichtanerkennungspflicht nach sich zieht, ist einer der rechtlichen Gründe für dic fehlende Durchsetzbarkeit dieses Rechtssatzes.

Daher wird eine Lösung des Konflikrs auch künftig an das Vorhandenscin eines entsprechenden politischen Willens einflußreicher Staaten geknüpft sein und eine Aufgabe für die politischen Organe der VN blciben. ${ }^{126}$ Die Gefahr besteht, daß die Staatenwelt den status quo Schritt für Schritt alkzeptiert und schließlich rechtlich anerkennt. Um so wichtiger erscheint es daher, den Konflikt in Ost-Timor durch Maßnahmen, wie z. B. die Vcrleihung des Friedensnobelpreises 1996 gemeinsam an den katholischen Bischof von Ost-Timor, Carlos Felipe Ximenes Belo und an den im Ausland lebenden Vizepräsidenten des "Nationalen Widerstandsrates " (CNRM) José Ramos Horta in das Bewußtsein der internationalen Öffentlichkeit zu rücken, um einen möglichen Konsolidierungsprozeß zu verhindern.

122 Laren\%, Mechodenlehre der Rechtswissenschaft (1979), S. 281 f.

123 Dahim/Delbrück/Wolfrum, Volkerrecht, Bd. I/1, S. 43.

124 Cassese (Fn. 13), S. 245 ; Hilpold (Fn. 16), S. 77.

125 Dahm/Delbrick/Wolfrum, (Fn. 123), S. $96 \mathrm{f}$

126 Cassese (Fn. 13), S. 344 f. 